\title{
Somatosensory evoked potentials and dopaminergic responsiveness to apomorphine and levodopa in parkinsonian patients
}

\author{
M. Miranda', J.L. Castillo ${ }^{2}$ and F. Araya ${ }^{2}$ \\ ${ }^{1}$ Neurology Unit, Félix Bulnes Hospital and ${ }^{2}$ Department of Neurological Sciences, Salvador \\ Hospital, University of Chile, Santiago, Chile
}

Correspondence to: M.Miranda Llewellyn Jones 1530, Depto 201, Providencia, Santiago, Chile

\begin{abstract}
Short-latency somatosensory evoked potentials (SSEPs) were recorded from 10 parkinsonian patients in 'off' and 'on' states induced by apomorphine and levodopa. The effects of apomorphine and long-term levodopa treatment on the frontal N30 component were assessed and compared with healthy controls. Nine of 10 patients tested with apomorphine showed a significant improvement $(p<0.01)$ in $\mathrm{N30}$ frontal component amplitude whereas in six of eight patients similarly assessed with levodopa we obtained a comparable improvement in SSEPs $(p<0.01)$. Parietal SSEPs remained unchanged. This normalization of frontal SSEPs was concomitant with the clinical response and in some patients preceded the motor response. No changes were obtained in control subjects. The improvement in N30 potential occurred regardless of disease duration or the presence of motor fluctuations. SSEPs may represent an objective approach for assessing the dopaminergic response and the fluctuations of motor disability in parkinsonian patients.
\end{abstract}

Keywords: Apomorphine - Levodopa - Motor fluctuations - Parkinson's disease - Somatosensory evoked potentials

\section{INTRODUCTION}

Short-latency somatosensory evoked potentials (SSEPs) to median nerve stimulation at the wrist are characterized in the frontal region by a wave called N30 (Desmedt and Cheron, 1980). Recent observations showed that abnormalities of the frontal N30 component correlate with dysfunction of neuronal circuits involved in movement disorders (Parkinson's disease, Huntington's disease, dystonia, progressive supranuclear palsy and MPTP-treated monkeys (Rossini et al., 1989; Onofrj et al., 1990; Abbruzzese et al., 1991; Relly et al., 1992; Topper et al., 1993). The origin of this frontal component is still a matter of controversy, but there is evidence that it is at least partially generated in a loop involving the motor supplementary area (SMA) and the related cortico-subcortical structures (Brinkman and Porter, 1983; Tanji and Kurata, 1983). Rossini et al. (1993), recently reported a potentiation of $\mathrm{N} 30$ frontal component amplitude in relation to improvement by apomorphine of parkinsonian symptoms. However, other recent studies by Maugiere et al. (1993) and Onofrj et al. (1995), failed to confirm this.

The clinical response to apomorphine and levodopa is identical in individual parkinsonian patients (Kempster et al., 1990; Rodriguez et al., 1994), as is the improvement in regional cerebral blood flow in the supplementary motor area (Jenkins et al., 1992; Rascol et al., 1994). It is interesting to determine therefore whether neurophysiological differences exist between apomorphine and levodopa within the cortical-subcortical motor loop.

We have studied changes in the N30 potential induced by both apomorphine and levodopa in a group of parkinsonian patients and its characteristics in a healthy control group.

\section{SUBJECTS AND METHODS}

After informed consent, 10 patients, six men and four women, mean age 61 years (range 54-74) were studied. The mean duration of disease was 4.5 years (range 1-17). All patients had idiopathic Parkinson's disease. The severity of the disease was scored according to the Hoehn-Yahr scale and the patients were classified as stage 1 (three patients), stage 2 (two patients), stage 3 (four patients) and stage 4 (one 
patient). Three patients had never been treated with levodopa or any antiparkinsonian drug ('de novo'), five showed daily motor fluctuations and two had stable Parkinson's disease after a mean 5.1 years (1-15) of levodopa treatment.

Parkinsonian patients were divided into two groups: one group included five patients with 5 or more years of disease duration (all of whom experienced motor fluctuations) and the other group five patients with less than 5 years of evolution (stable or minimal fluctuations).

We assessed the patients in the morning and in the treated patients all dopaminergic drugs were interrupted for at least $12 \mathrm{~h}$ before the apomorphine test. Domperidone was given orally to every patient at 90 $\mathrm{mg} /$ day for 2 days before the apomorphine challenge to prevent adverse effects. Apomorphine chloride $(5 \mathrm{mg} / \mathrm{ml})$ was given subcutaneously using the protocol proposed by Hughes et al. (1990), starting with 1.5 $\mathrm{mg}$ and increasing to a maximum of $5 \mathrm{mg}$ via an insulin syringe in the arm, until a positive response was obtained or adverse reactions occurred.

Motor function was asssessed at baseline (time 0) and $10 \mathrm{~min}$ after each doses of apomorphine. Motor tests included finger tapping for $15 \mathrm{~s}$, time required to walk 12 meters and the Webster (1968) scale. The apomorphine test was considered positive if there was greater than $15 \%$ improvement in finger tapping, more than $25 \%$ in walking time and improvement in three or more parameters on the Webster scale.

SSEPs were recorded using silver disk electroencephalogram electrodes placed contralaterally to the stimulus site (parietal: $7 \mathrm{~cm}$ lateral to $\mathrm{Cz}$ and $2 \mathrm{~cm}$ behind the $\mathrm{Cz}-$ ear line, frontal: $5 \mathrm{~cm}$ frontal to $\mathrm{Cz}$ and $5 \mathrm{~cm}$ lateral to the midline). A reference electrode was placed on Fpz. The median nerve of the clinically more affected side was stimulated at the wrist with electrical square wave pulses of $0.2 \mathrm{~ms}$ duration. Stimulus intensity was set to produce a visible thumb twitch at $4 \mathrm{~Hz}$ repetition rate.

Patients were sitting comfortably on a chair and were encouraged to relax as much as possible while avoiding falling asleep. Two hundred to 300 'artifactfree' responses were filtered $(10 \mathrm{~Hz}-3 \mathrm{KHz})$ and averaged with an analysis time of $50 \mathrm{~ms}$. At least two traces were obtained and superimposed to check for their reproducibility. SSEPs were recorded at time 0 and after 10-15 min of each dose of apomorphine.

The following components were studied: peak latencies of the parietal wave N20 and of the frontal wave N30, peak to peak amplitudes of the parietal N20 (P14-N20) and of the frontal N30 (P20-N30).

Two healthy volunteers (52 and 56 years old) were taken as controls using the same neurophysiological and pharmacological apomorphine protocol. A group of six healthy controls (mean age 61 years) were considered to define the spontaneous variability of the latency/amplitude of SSEPs with time (60 $\mathrm{min})$. SSEPs amplitude varied between 0.37 to $1.00 \mu \mathrm{V}$ and we considered such a range to define the changes in patients as significant.

Eight of the 10 patients were reassessed clinically and electrophysiologically, during levodopa treatment when in an 'on' state, aproximately one month after the initial challenge with apomorphine.

Statistical analysis of SSEPs data and clinical parameters was performed using the Wilcoxon matchedpairs signed rank test for intra-group comparisons of the means of different variables.

\section{RESULTS}

All patients showed clinical improvement with apomorphine and most of them had a clinical response with a mean dose of $3 \mathrm{mg}$ apomorphine (range 1.5-5 $\mathrm{mg}$ ). They developed an 'on' phase 10-20 min after injection of the effective dose.

TABLE I. Mean latencies and amplitudes of parietal and frontal potentials in the basal condition ('off state') and after apomorphine or levadopa.

\begin{tabular}{llrl}
\hline SSEP & Treatment & Mean & S.D. \\
\hline $\mathrm{N}-20(\mathrm{~ms})$ & Basal & 18.85 & 1.27 \\
& Apomorphine & 18.91 & 1.33 \\
$\mathrm{~N}-30(\mathrm{~ms})$ & Basal & 27.65 & 2.08 \\
& Apomorphine & 28.41 & 1.95 \\
$\mathrm{P} 14-\mathrm{N} 20(\mu \mathrm{V})$ & Basal & 2.19 & 0.72 \\
& Apomorphine & 2.13 & 0.84 \\
$\mathrm{P} 20-\mathrm{N} 30(\mu \mathrm{V})$ & Basal & 1.52 & 0.85 \\
& Apomorphine & $2.64^{1}$ & 1.36 \\
& Levodopa & 2.32 & 1.31 \\
\hline
\end{tabular}

${ }^{1}$ Frontal N30 component (P20-N30) significantly improved after apomorphine and levodopa $(p<0.01)$.

The neurophysiological findings are summarized in Table I. Nine of 10 patients showed a significant increase $(p<0.01)$ in the frontal N30 component amplitude following apomorphine (Fig. 1). It is noteworthy that the patient who had no increase in amplitude in SSEPs (patient 6), had a better morphological configuration of the N30 wave. In six patients the change in the amplitude of potential preceded the clinical response (before $10 \mathrm{~min}$ ), and in three it appeared to be simultaneous. No significant changes of SSEPs were observed in the healthy controls following apomorphine administration.

Parietal SSEPs (N20) were not significantly influenced by apomorphine. In six of eight patients who 


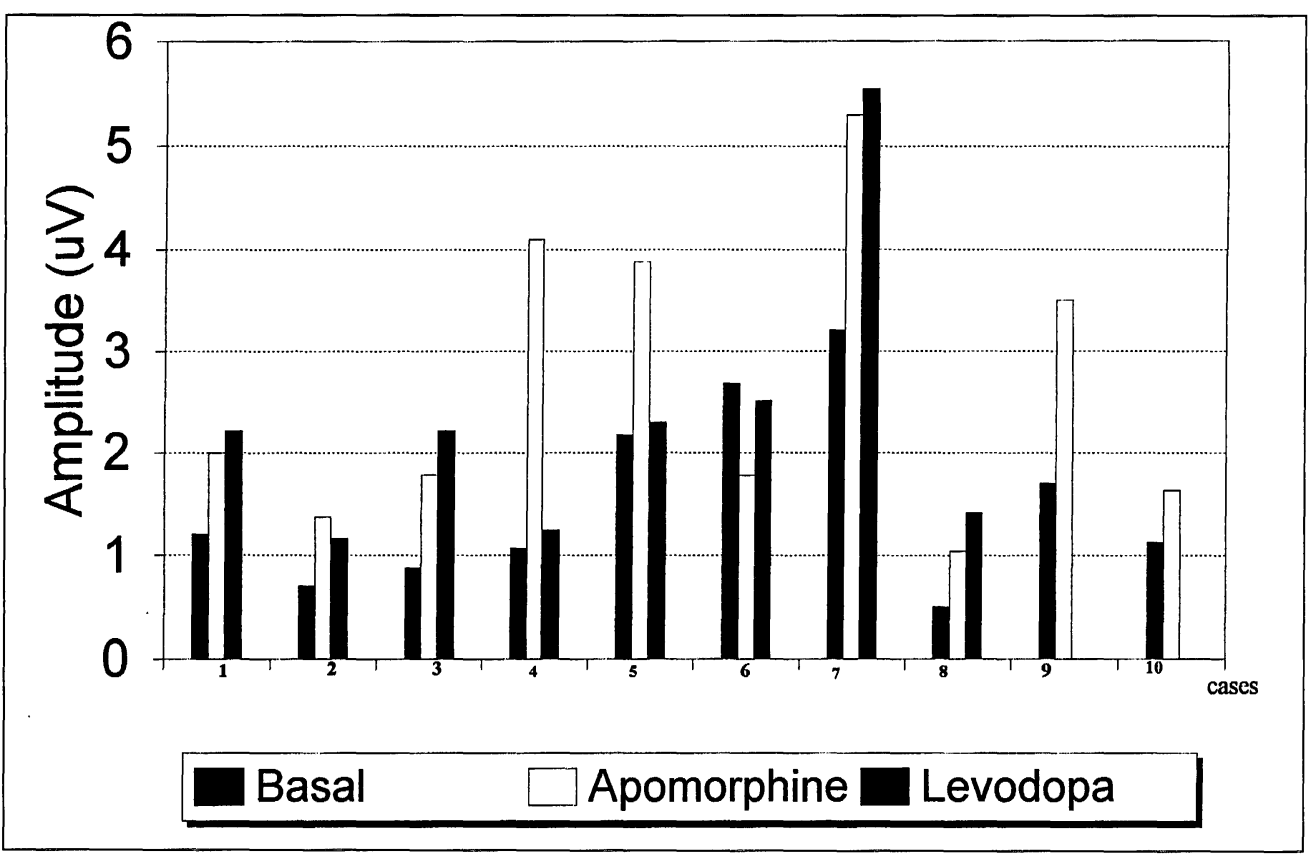

FIG 1. N30 potential amplitude in the 'off' state and after using apomorphine and levodopa in 10 parkinsonian patients. Two patients were tested only with apomorphine (patients 9 and 10). Both apomorphine and levodopa induced normalization of the N30 potential amplitude except in one patient (patient 6).
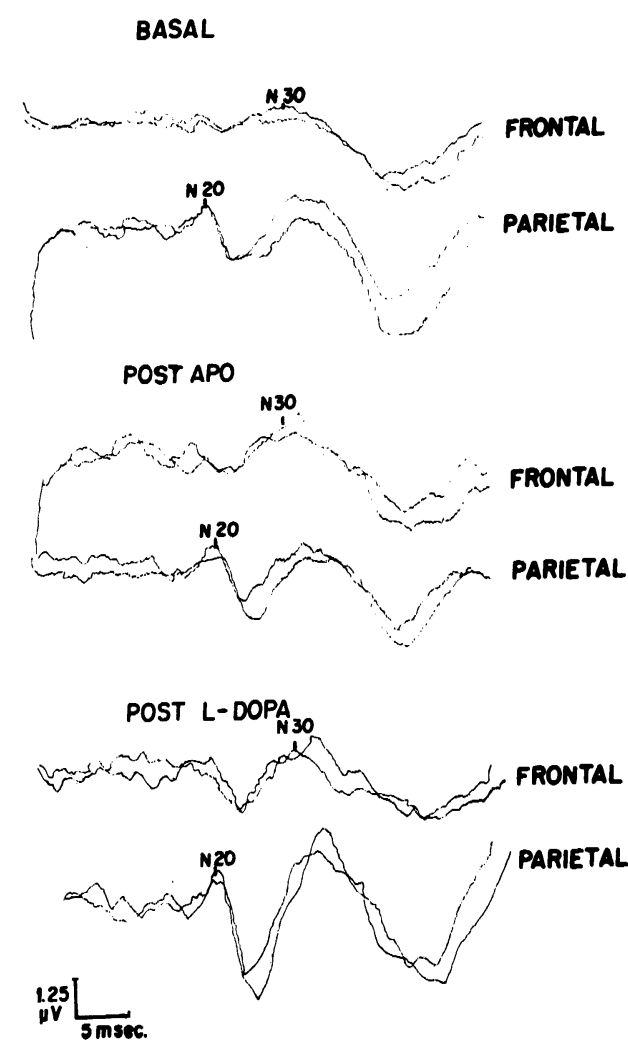

FIG. 2. Patient 3, a 67-year-old man. In the basal condition a very low amplitude frontal potential is evoked. Twenty min following subcutaneous apomorphine, a clear-cut frontal N30 was recorded. After using levodopa for a month, the $\mathrm{N} 30$ potential obstained was very similar. were reassesssed on levodopa in the 'on' state, we found a clear improvement in the amplitude of N30 potential $(p<0.01)$ and again parietal SSEPs $(\mathrm{N} 20)$ remained unchanged.

Apomorphine and levodopa therefore both induced normalization of a reduced frontal N30 component in a similar fashion but with differing magnitude responses as shown in Fig.2.

The mean basal N30 amplitude of control subjects (2.55 $\mu \mathrm{V}$, S.D. 1.72) was very similar to the mean N30 amplitude of the parkinsonian patients in the 'on' state induced by apomorphine $(2.64 \mu \mathrm{V}$, S.D. 1.36).

The finger tapping test showed the greatest increase $(p<0.01)$ in the clinical parameters of motor response.

The restoration in $\mathrm{N} 30$ frontal component amplitude induced by apomorphine and levodopa occurred regardless of disease duration or the presence of motor fluctuations, although the magnitude of SSEP improvement was more pronounced in the more severly affected patients. However, this difference was not statistically significant.

Although the clinical response was similar for levodopa and apomorphine in individual parkinsonian patients, the electrophysiological response was less pronounced with levodopa.

Side-effects were noted in six cases after apomorphine. They included vomiting (three), yawning (two) and hypotension (one), but were not severe enough to interrupt the recording. 


\section{DISCUSSION}

Our findings are broadly in accordance with Rossini et al.'s (1993) study, where a clear improvement in the frontal N30 component induced by apomorphine occurred in 22 of 32 patients. We also found comparable changes with levodopa. We observed that in parkinsonian patients the reduced baseline N30 frontal amplitude was restored to normal after using apomorphine or levodopa. However, our results are at variance with the findings of Maugiere et al. (1993), who described no changes in SSEPs after chronic apomorphine therapy in parkinsonian patients and also with the study by Onofrj et al. (1995) who found no changes after acute challenge with levodopa and chronic dopaminergic therapy. We have no clear explanation for these different results.

We found a clear correlation between the changes in the 'off' to the 'on' state and the electrophysiological response, with the SSEPs changes even preceding the clinical improvement in some patients.

By means of SSEPs it is possible therefore to confirm neurophysiologically the similar biological response of apomorphine and levodopa in patients with different stages of disease. This observation has also been confimed clinically and in SPECT studies (Kempster et al., 1990; Jenkins et al., 1992; Rascol et al., 1994; Rodriguez et al., 1994).

We agree with Rossini et al. (1993) that SSEPs can be an objective method for assessing the dopaminergic response, and might complement the apomorphine test, by facilitating its interpretation in borderline responses.

N30 potential has been postulated to be generated in the neuronal circuit involving supplementary motor area, basal ganglia, primary motor cortex and ventro-lateral thalamic nuclei (Brinkman and Porter, 1983; Tanji and Kurata, 1983). PET and SPECT studies have shown clear improvement in regional cerebral blood flow in the supplementary motor area in parkinsonian patients treated with apomorphine as well as with levodopa (Jenkins et al., 1992; Rascol et al. 1994). The regional cerebral blood flow increase in the SMA probably reflects an improvement in the underlying neuronal activity. This is related to the dopaminergic effect of these drugs in the basal ganglia improving the pallido-thalamic-cortical outflow (Delong, 1990).

These observations support the hypothesis that a functional deafferentation of the SMA plays an important role in the pathophysiology of akinesia (Jenkins et al., 1992; Rodriguez et al., 1994). Our study suggests that the normalization of N30 frontal component might be another expression of the restoration of SMA activity induced by apomorphine and levodopa.

Recently, Remy et al. (1994) described a significant increase of activity of SMA in a PET study in normal subjects in difficult motor control tasks such as flexing and extending their middle fingers triggered by an external cue. This supports the notion that SMA is involved in initiating movements triggered by sensory cues. It may be, therefore, that assessment of motor function with finger tapping (this tapping test is well known to be reliable in the evaluation of dopaminergic response), enhances SMA activation and makes SSEP changes easier to record.

The similar SSEP response obtained with apomorphine and levodopa in individual parkinsonian patients at different stages, further supports the hypothesis that postsynaptic mechanisms play an important role in the origin of motor fluctuations in Parkinson's disease (Kempster et al., 1990; Rodriguez et al., 1994).

\section{Acknowledgements}

We wish to thank Dr Andrew Lees of the Department of Neurology, University College Hospital, London, for critically reviewing the manuscript.

\section{REFERENCES}

Abbruzzese G, Tabaton M, Morena M, Dallagata D and Favale E (1991) Motor and sensory evoked potentials in progressive supranuclear palsy. Movement Disorders, 6, 49-54.

Brinkman C and Porter R (1983) Supplementary motor area and premotor area of monkey cerebral coretex: functional organization of single neurons during performance of a learned movement. In: Motor Control Mechanisms in Health and Disease. Advances in Neurology, vol 39, Ed. JE Desmedt, pp. 393-420. Raven Press, New York.

Delong M (1990) Primate models of movement disorders of basal ganglia origin. Trends in Neuroscience, 13, 381-385.

Desmedt JE and Cheron G (1980) Somatosensory evoked potentials to finger stimulation in healthy octogenarians and in young adults: wave forms, scalp topography and transit times of parietal and frontal components. Electroencephalography and clinical Neurophysiology, 50, 404-425.

Hughes AJ, Lees AJ and Stern GM (1990) Apomorphine test to predict dopaminergic responsiveness in parkinsonian syndromes. Lancet, 336, 32-34.

Jenkins IH, Fernandez W and Playford ED (1992) Impaired activation of the supplementary motor area in Parkinson's disease is reversed when akinesia is treated with apomorphine. Annals of Neurology, 32, 749-757.

Kempster PA, Frankel JP, Stern GM and Lees AJ (1990) Comparison of motor response to apomorphine and levodopa in Parkinson's disease. Journal of Neurology, Neurosurgery and Psychiatry, 53, 1004-1007. 
Maugiere F, Broussolle E and Isnard J (1993) Apomorphine-induced relief of the akinetic-rigid syndrome and early median nerve somatosensory evoked potentials in Parkinson's disease. Electroencephalography and clinical Neurophysiology, 88, 243-254.

Onofrj M, Ghilardi MF, Basciani M, Martinez T and Glover A (1990) Attenuation of the early anterior negativity of median nerve somatosensory evoked potential in the MPTP-treated monkey. Clinical Neurophysiology, 20, 283-293.

Onofrj M, Fulgente G, Malatesta et al. (1995) The Abormality of N30 somatosensory evoked potential in idiopathic Parkinson's disease is unrelated to disease stage or clinical scores and insensitive to dopamine manipulations. Movement Disorders, 10, 71-80.

Rascol O, Sabatini U, Chollet F et al. (1994) Normal activation of the supplementary motor area in patients with Parkinson's disease undergoing long-term treatment with levodopa. Journal of Neurology, Neurosurgery and Psychiatry, 57, 567-571.

Relly JA, Hallet M, Cohen LG, Tarkka IM and Dang N (1992) The N30 component of somatosensory evoked potentials in patients with distonia. Electroencephalography and Clinical Neurophysiology, 84, 243-247.

Remy P, Zilbovicius M and Leroy-Willig A (1994) Movement and task-related activations of motor cortical areas: a positron emission tomographic study. Annals of Neurology, 36, 19-26.
Rodriguez M, Lera G, Vaamonde J, Luquin MR and Obeso JA (1994) Motor response to apomorphine and levodopa in asymmetric Parkinson's disease. Journal of Neurology, Neurosurgery and Psychiatry, 57, 562-566.

Rossini PM, Babiloni F, Bernardi G, Cecchi L, Johnson PB and Malentacca A (1989) Abnormalities of shortlatency somatosensory evoked potentials in parkinsonian patients. Electroencephalography and Clinical Neurophysiology, 74, 277-289.

Rossini PM, Traversa R, Boccasena P et al. (1993) Parkinson's disease and somatosensory evoked potentials: Apomorphine-induced transient potentiation of frontal components. Neurology, 43, 2495-2500.

Tanji I and Kurata K (1983) Functional organization of the supplementary motor area. In: Motor Control Mechanisms in Health and Disease. Advances in Neurology, vol 39 Ed. JE Desmedt. pp 421-431. Raven Press, New York.

Topper R, Schwarz M, Podoll K, Danzer F and Roth J (1993) Absence of frontal somatosensory evoked potentials in Huntington's disease. Brain, 116, 87-101.

Webster DD (1968) Critical analysis of the disability in Parkinson's disease. Modern Treatments, 5, 257-282.

(Received 15 March 1996; accepted 10 October 1996) 


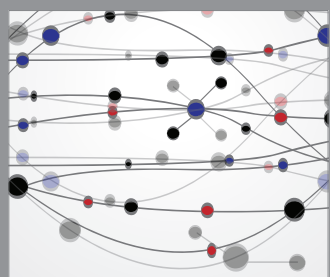

The Scientific World Journal
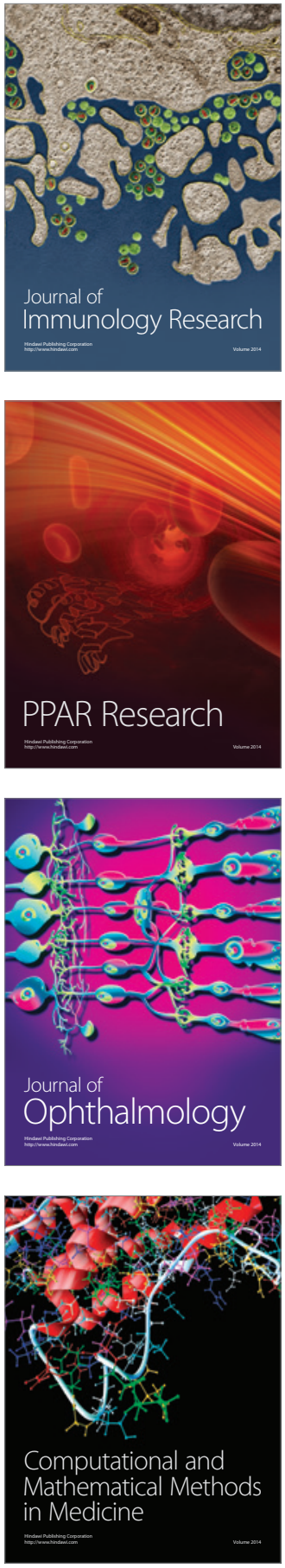

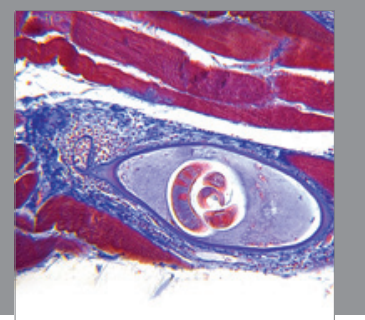

Gastroenterology

Research and Practice
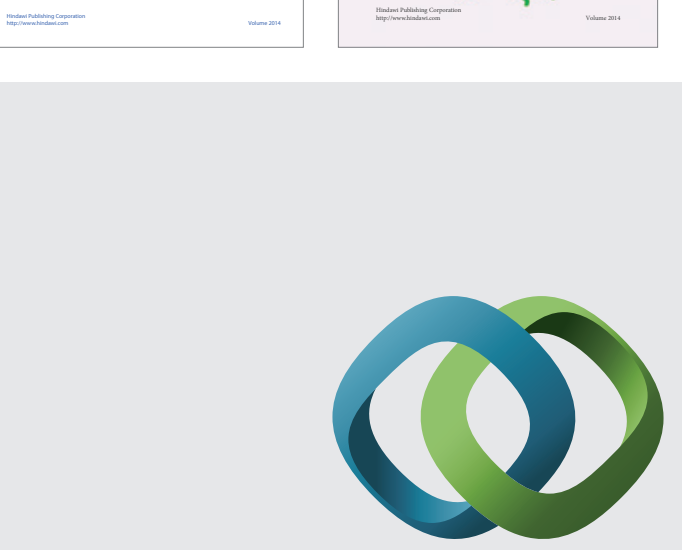

\section{Hindawi}

Submit your manuscripts at

http://www.hindawi.com
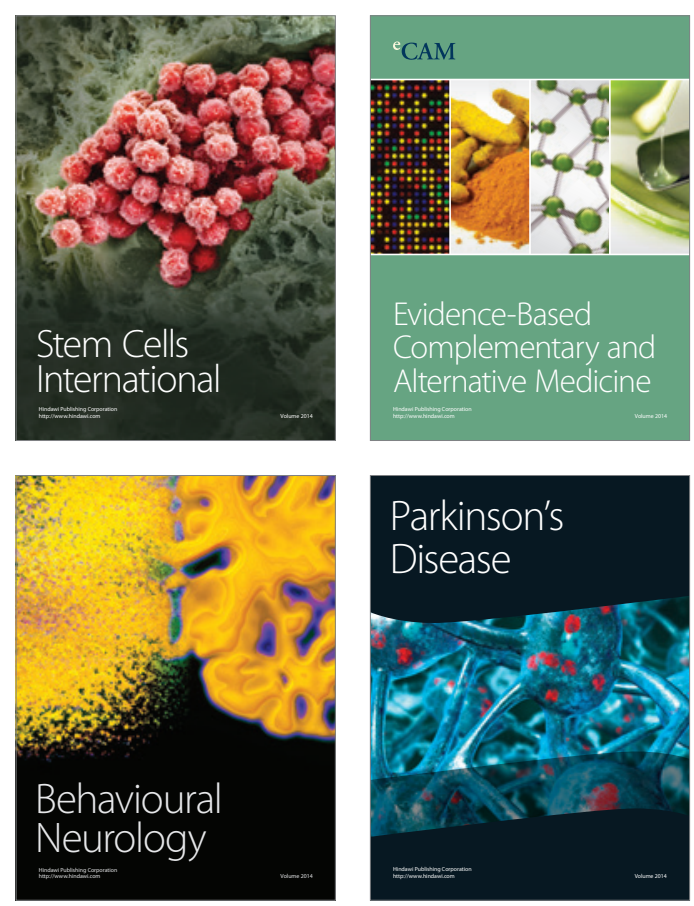

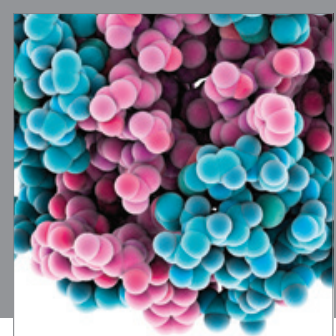

Journal of
Diabetes Research

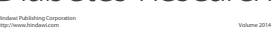

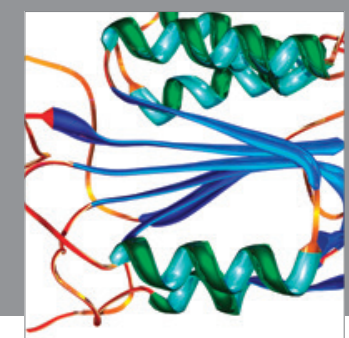

Disease Markers
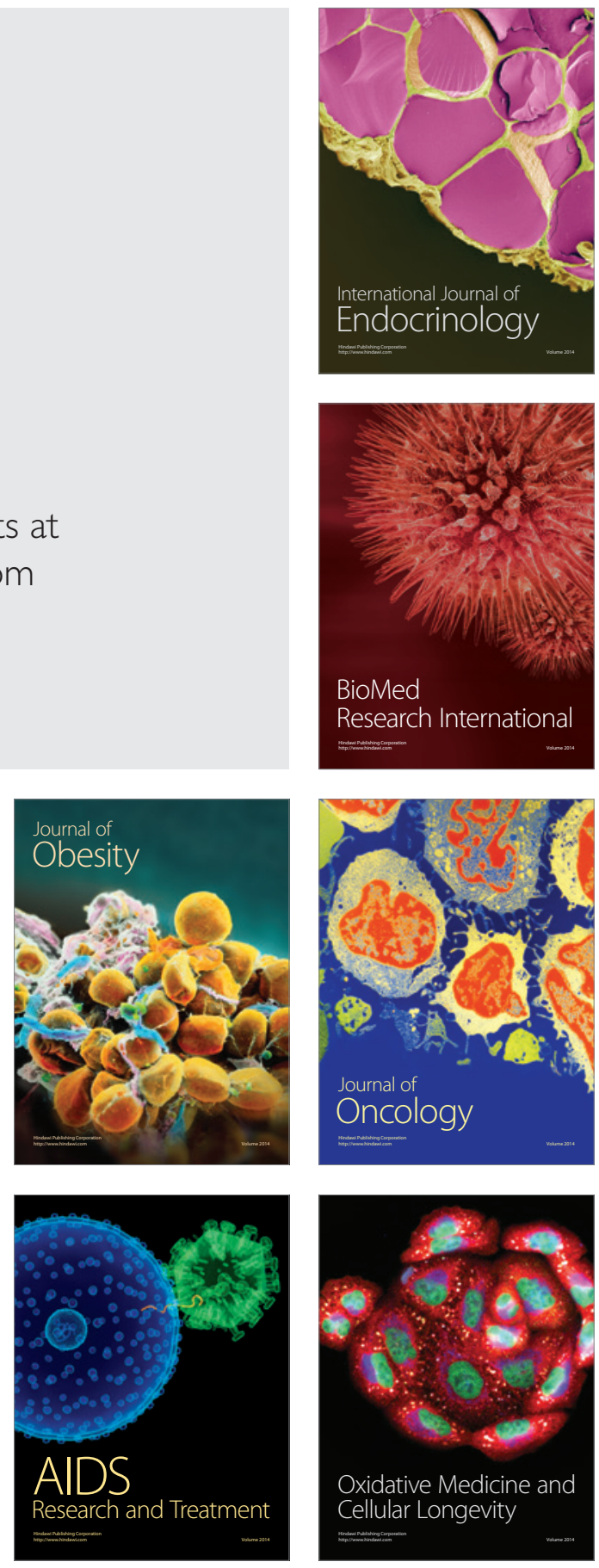